\title{
S\&I Public Health Reporting Initiative: Improving Standardization of Surveillance
}

\author{
Michael Coletta*1, Nikolay Lipskiy ${ }^{2}$, David Birnbaum ${ }^{3}$ and John Abellera ${ }^{2}$ \\ ${ }^{1} \mathrm{NACCHO}$, Washington, DC, USA; ${ }^{2} \mathrm{CDC}$, Atlanta, GA, USA; ${ }^{3}$ Washington State Department of Health, Olympia, WA, USA
}

\section{Objective}

The objective of this panel is to inform the ISDS community of the progress made in the Standards \& Interoperability (S\&I) Framework Public Health Reporting Initiative (PHRI). Also, it will provide some context of how the initiative will likely affect biosurveillance reporting in Meaningful Use Stage 3 and future harmonization of data standards requirements for public health reporting.

\section{Introduction}

The S\&I Framework is an Office of National Coordinator (ONC) initiative designed to support individual working groups who focus on a specific interoperability challenge. One of these working groups within the S\&I Framework is the PHRI, which is using the S\&I Framework as a platform for a community-led project focused on simplifying public health reporting and ensuring EHR interoperability with public health information systems. PHRI hopes to create a new public health reporting objective for Meaningful Use Stage 3 that is broader than the current program-specific objectives and will lay the ground work for all public health reporting in the future. To date, the initiative received over 30 descriptions of different types of public health reporting that were then grouped into 5 domain categories. Each domain category was decomposed into component elements and commonalities were identified. The PHRI is now working to reconstruct a single model of public health reporting through a consensus process that will soon lead to a pilot demonstration of the most ready reporting types. This panel will outline progress, challenges, and next steps of the initiative as well as describe how the initiative may affect a standard language for biosurveillance reporting.

\section{Methods}

Michael Coletta will provide an introduction and background of the S\&I PHRI. He will describe how the PHRI intends to impact reporting in a way that is universal and helpful to both HIT vendors and public health programs.
Nikolay Lipskiy will provide an understanding of the ground breaking nature of collaboration and harmonization that is occurring across public health programs. He will describe the data harmonization process, outcomes, and hopes for the future of this work.

David Birnbaum has been a very active member of PHRI and has consistently advocated for the inclusion of Healthcare Associated Infections (HAI) reporting in Meaningful Use as a model. David has been representing one of the largest user communities among those farthest along toward automated uploading of data to public health agencies. He will describe the opportunities and challenges of this initiative from the perspective of a participant representing an already highly evolved reporting system (CDC's National Healthcare Safety Network system).

John Abellera has been the steward of the communicable disease reporting user story for the PHRI. He will describe the current challenges to reporting and how the PHRI proposed changes could improve communicable disease reporting efforts.

This will be followed by an open discussion with the audience intended to elicit reactions regarding an eventual consolidation from individual report specific specification documents to one core report specification across public health reporting programs which is then supplemented with both program specific specifications and a limited number of implementation specific specifications.

\section{Results}

Plan to engage audience: Have a prepared list of questions to pose to the audience for reactions and discussion (to be supplied if participation is low).

\section{Keywords}

Standards; Interoperability; Meaningful Use; Reporting; Stage 3

*Michael Coletta

E-mail: mcoletta@naccho.org 